\title{
Solar activity - past, present, future
}

\author{
Leif Svalgaard ${ }^{\mathrm{a}, *}$ \\ W.W. Hansen Experimental Physics Laboratory, Stanford University, CA 94305, California, USA \\ *Corresponding author: leif@leif.org
}

Received 31 January 2013 / Accepted 30 April 2013

\begin{abstract}
As our civilization depends increasingly on space-borne assets and on a delicate and vulnerable earth-bound infrastructure, solar activity and its potential impact becomes of increasing importance and relevance. In his famous paper on the Maunder Minimum, Eddy (1976) introduced the notion that the Sun is a variable star on long time scales. After the recent decade of vigorous research based on cosmic ray and sunspot data as well as on geomagnetic activity, an emerging consensus reconstruction of solar wind magnetic field strength has been forged for the last century. The consensus reconstruction shows reasonable agreement among the various reconstructions of solar wind magnetic field the past $\sim 170$ years. New magnetic indices open further possibilities for the exploitation of historic data. The solar wind is a direct result of solar magnetic activity providing an important link to the effects on the Earth's environment. Reassessment of the sunspot series (no Modern Grand Maximum) and new reconstructions of Total Solar Irradiance also contribute to our improved knowledge (or at least best guess) of the environment of the Earth System, with obvious implications for management of space-based technological assets or, perhaps, even climate. Several lines of evidence suggest that the Sun is entering a period of low activity, perhaps even a Grand Minimum. Average space weather might be "milder" with decreased solar activity, but the extreme events that dominate technological effects are not expected to disappear. Prediction of solar activity has a poor track record, but the progression of the current Cycle 24 is in accordance with its behavior predicted from the evolution of the solar polar fields, so perhaps there is hope.
\end{abstract}

Key words. solar activity - cycle prediction - time variation - historical data - solar wind

\section{Introduction}

Solar activity is the result of solar magnetic fields. If our Sun had no magnetic field it would be as dull as models of stellar constitution proscribe and we would not have this conference. The magnetic field makes the Sun interesting, which before the development of our technological civilization was of little consequence, but that the Sun is a variable magnetic star is today of immense practical importance; in fact, a potential danger to our modern way of life. Increasingly, our civilization depends on environmental conditions, communication devices, and infrastructures that are vulnerable to solar magnetic variability (NRC 2008). The famous Carrington Event in 1859 (e.g., Cliver \& Svalgaard 2004) can be said to mark the birth of this concern, although the technological effects, some even damaging with attendant economics loss, of geomagnetic disturbances (accompanied by brilliant aurorae) on the nascent telegraph communication capability, were already noted more than a decade before.

To assess the impact of solar activity and the chances of effective mitigation of its effect we need to monitor and understand not only current space weather, but also space climatology: what is the equivalent of a "hundred-year flood"? Direct telescopic observation of solar activity, of course, begins with the discovery 402 years ago of sunspots. Our understanding of that historical record forms the basis for interpreting the indirect evidence both from natural archives (e.g., ${ }^{10} \mathrm{Be}$ from ice cores) and human naked-eye observations (aurorae, 日誌 [ri-zhi] blemishes on the sun) stretching much farther back in time.

\footnotetext{
a Details of the analyses and related talks and papers can be found at http://www.leif.org/research
}

\section{The sunspot record(s)}

The historical sunspot record was first assembled by Rudolf Wolf in 1850s and has been continued by Wolf and his successors followed by a more "institutionalized" approach later in the 20th century until today and hopefully beyond. Wolf's original definition of the Relative Sunspot Number for a given day as $R=10$ Number of Groups + Number of Spots visible on the solar disk has stood the test of time and recognizes (in Wolf's own words) that the emergence of a coherent group of spots (an active region) is much more important than the addition of yet a few spots to an existing group. The factor of 10 has also turned out to be a good choice as historically a group contained on average ten spots.

A fundamental problem is the homogeneity of the series, that is: does a relative sunspot number of 100 mean the same level of solar activity today as it did in 1938, in 1872, or in 1739 ? And what is a useful definition of "solar activity" anyway? From the viewpoint of solar effects on our technological infrastructure, the solar wind - the ever-present expanding outer atmosphere of the Sun - is perhaps the most relevant element, although bursts of highly energetic particles and radiation also degrade devices and spacecraft and threaten humans in space. Almost all solar indices and solar wind quantities show a relationship with the Relative Sunspot Number (SSN), so homogeneity and proper calibration of the SSN become of utmost importance.

Hoyt et al. (1994) in a series of papers at the centenary of Wolf's death asked "Do we have the correct reconstruction of solar activity?" and proposed to answer the question in the negative. A heroic effort from an extensive search of archives 


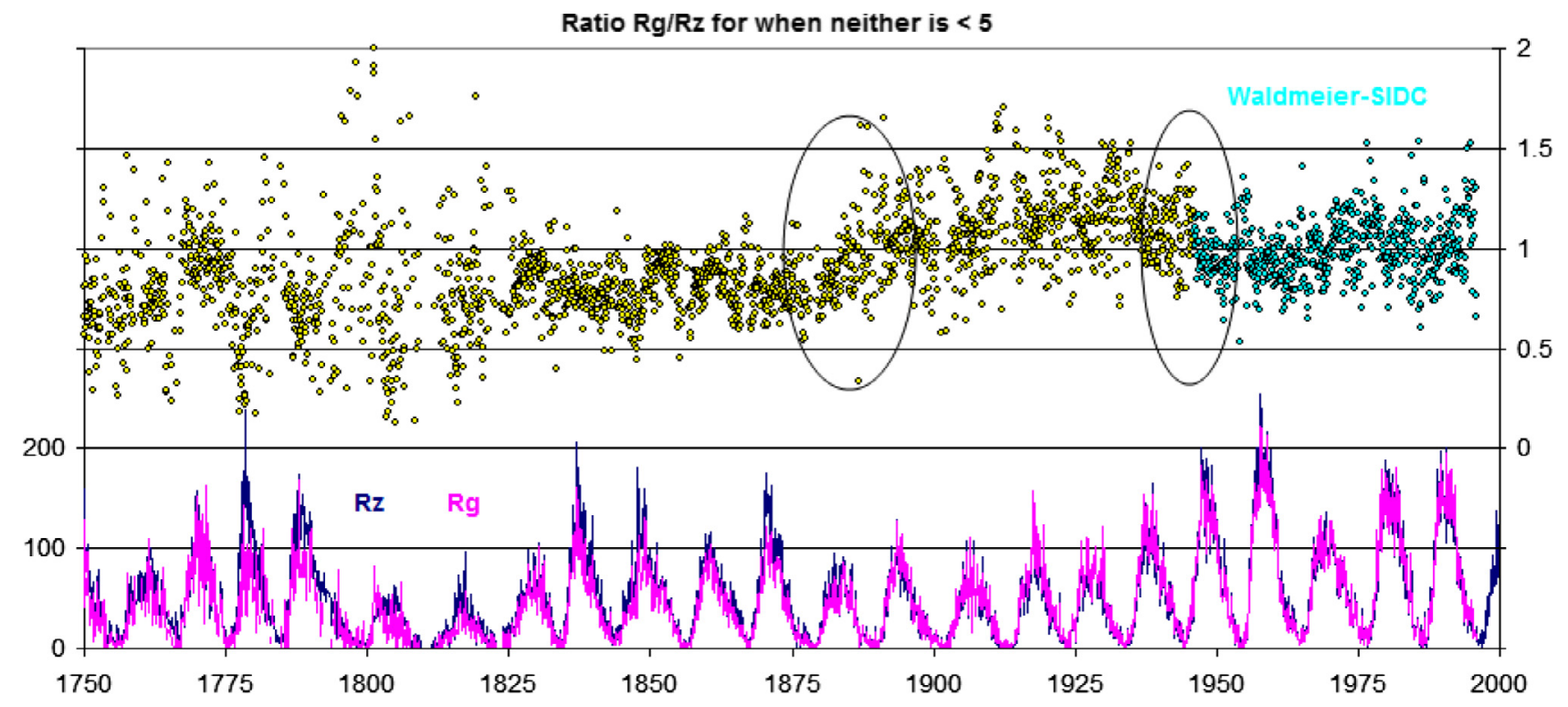

Fig. 1. Ratio between monthly values of the Group Sunspot Number $(\mathrm{Rg})$ and the Wolf (Zürich, International) Sunspot Number.

and primary sources yielded $\sim 350,000$ observations, many not available to Wolf, covering the interval 1610-1993. An earlier study showed that the ratio of individual spots to groups is nearly a constant. Theoretical arguments (Schaefer 1993) showed that Wolf's Relative Sunspot Number may be set equal to a constant times the number of sunspot groups, so an index based solely on the number of sunspot groups can simulate the Wolf SSN. Hoyt and Schatten called this index the Group Sunspot Number (GSN) and found that it appears that solar activity has steadily increased since 1700 to what might be called a Modern Grand Maximum in the latter part of the 20th century. Before $~ 1885$ the GSN is significantly smaller than the Wolf SSN that does not support the idea of a Modern Grand Maximum. This discrepancy is not satisfactory and must be resolved so solar-terrestrial researchers have a stable and unique dataset to work with.

Recognizing the need to resolve this issue, a number of workshops on the calibration of the sunspot number have been sponsored by the National Solar Observatory (NSO), the Royal Observatory of Belgium (ROB), and the Air Force Research Laboratory (AFRL) as an effort to provide the solar community with a vetted long-term (single) sunspot number series and the tools to keep it on track. The first workshop was held at Sunspot, New Mexico (yes, there is such a place) in September 2011, followed by a second workshop in Brussels in May 2012 and a third meeting in in Tucson, AZ, in 2013 with a final meeting in Locarno, Switzerland, in 2014. We are considering a special Topical Issue of Solar Physics for the eventual joint publication of the SSN series and the accompanying historical, procedural, and scientific papers. In this paper, I'll report on the progress made so far.

An efficient way of comparing the Wolf Sunspot Number and the Group Sunspot Number is to plot the ratio between them as shown in Figure 1. That removes most of the solar cycle variation and will show obvious discontinuities caused by non-solar related changes in the calibration. Figure 1 shows two clear discontinuities, one near 1945 and one near 1885.

Explaining those will go a long way to resolve the differences between the two series. We shall show that the 1945 discontinuity is a problem with the Wolf Number while the 1885 discontinuity is a problem with the Group Number. When both problems are corrected, there is no longer a significant difference between the two series.

\section{The Waldmeier discontinuity}

Some time in the 1940s the observers in Zürich began to weight sunspots when counting them. The director of the Zürich Observatory, Max Waldmeier described (Waldmeier 1968) the procedure thus "A spot like a fine point is counted as one spot; a larger spot, but still without penumbra, gets the statistical weight 2 , a smallish spot with penumbra gets 3 , and a larger one gets 5 ". This weighting increases the spot count by $45 \%$ on average and, since the spot count is half of the Relative Number, the SSN by approximately half that. The Locarno Station in Southern Switzerland has since 1957 served as an auxiliary site for Zürich (as the weather on opposite sides of the Alps often is complementary) and is still today the reference station for the modern sunspot number maintained by SIDC in Brussels, as all other observers are normalized to Locarno's count. Locarno is still weighting the spots according to Waldmeier's prescription, so the weighting carries over fully into the current sunspot number. Normalization is done by applying a factor, $k$, in the formula for the Relative Sunspot Number $R=k(10 G+S)$ such that different observers of the number of groups, $G$, and the number of spots, $S$, arrive at the same relative number, $R$. The $k$-factor depends on several things: telescope aperture and magnification, observer acuity, atmospheric seeing, and the precise way spots are recognized and grouped. The Zürich observers after Wolf chose to count all spots that were visible, while Wolf did not include the smallest spots near the limit of detectability, in order to be compatible with Schwabe's observations. Consequently, a $k$-factor of 0.6 was, at first, empirically determined and later simply adopted to reduce the sunspot number to the original Wolf scale.

Figure 2 shows the effect of weighting using a typical drawing from Locarno. There is also a small contribution from an improved classification of sunspot groups introduced at about the same time. The combined net effect is to increase the sunspot number since $\sim 1945$ by $\sim 20 \%$. This explains the discontinuity in the ratio GSN/SSN at that time. A strong check and confirmation of the effect of the weighting have been carried 


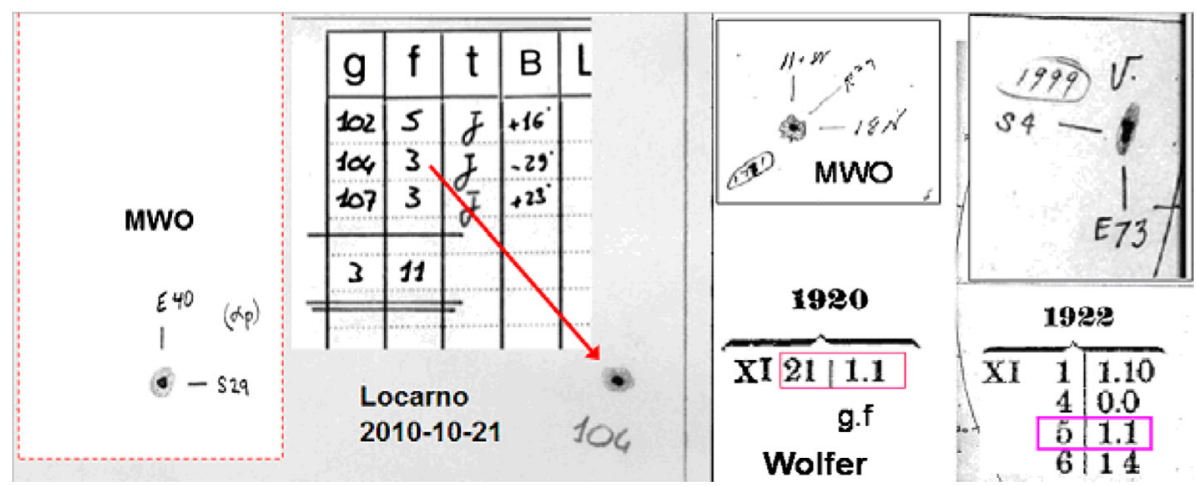

Fig. 2. Left: part of a drawing made at Locarno, showing that the spot with penumbra designated 104 was counted three times (weight 3 ); the inset shows the same spot from Mt. Wilson. Right: two spots with the same area on drawings from Mt. Wilson and counted with weight 1 by Wolfer, in each case as one group with one spot (Wolf's notation: groups.spots $=1.1$ ), as the group was the only group on the disk.

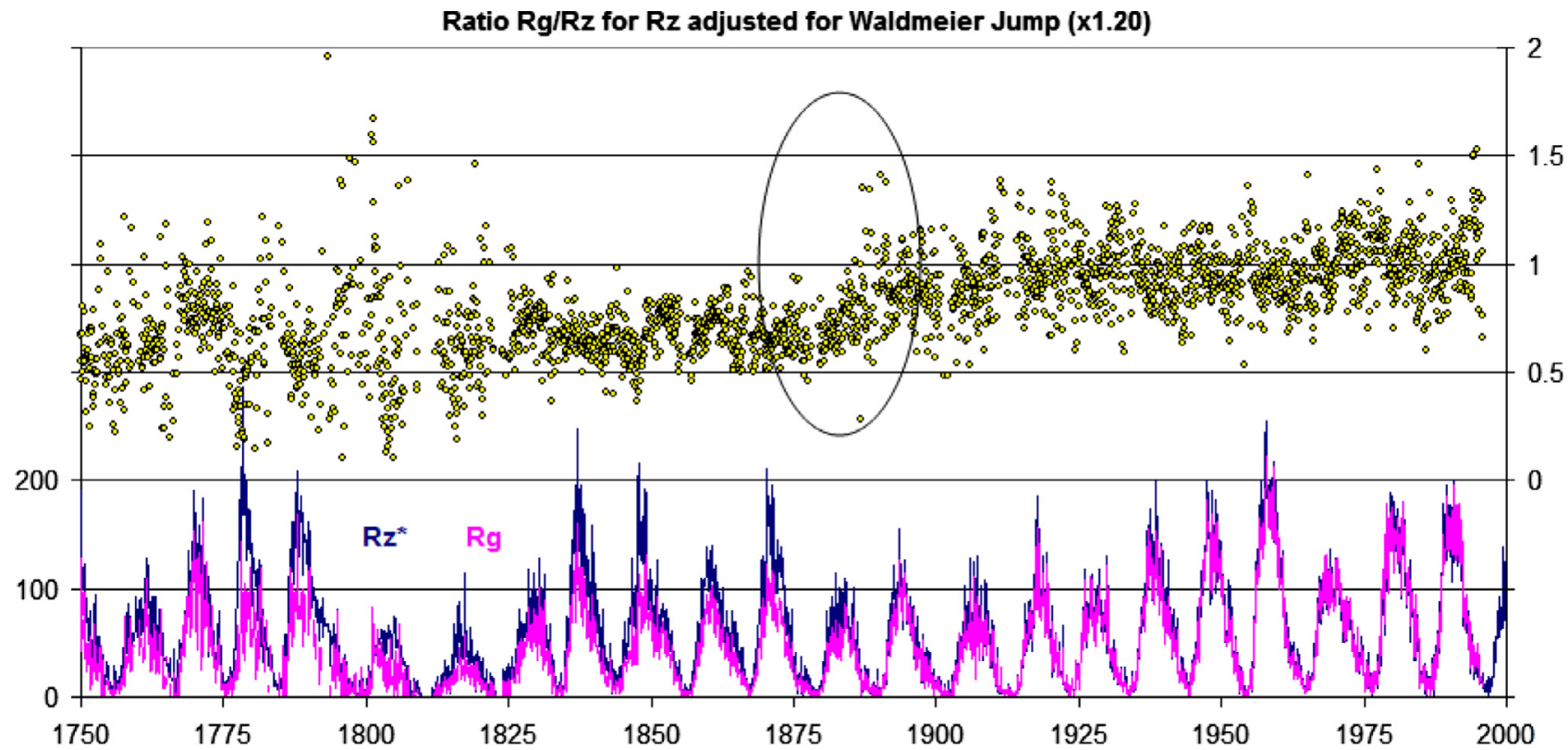

Fig. 3. Removing the effect of weighting after 1945 removes the discontinuity in 1945 . There remains the discrepancy around 1885 . The lower panel shows the corrected SSN (Rz) in blue and the GSN in pink. Note that the corrected SSN reaches about the same level in each century.

out the past year by the Locarno observers, counting both with and without weighting, so the magnitude of the effect of the weighting is now established and is no longer an open issue. The effect of the weighting turns out to be almost independent of solar activity, so a simple corrective action would be to multiply all numbers before 1945 by 1.20 . Figure 3 shows the result of the correction. Such "wholesale" correction is not without precedent. In 1861 Wolf published his first long list of the SSN covering the years 1749-1860. Around 1875, Wolf increased all the numbers on the 1861 list before 1848 by $25 \%$, based on measurements of "the magnetic needle", which we'll hear more about later in this paper.

\section{Group sunspot number calibration}

Hoyt and Schatten (H\&S) quickly found that the constant that converted a group count to a simulated Wolf Relative Number was not a constant at all, but varied with the observer, and was, in effect, a $k$-factor to be applied to the number of groups. A decision must be made as to which observer should have a $k$-factor of unity. H\&S chose the "Helio-Photographic Results" tabulations from Royal Greenwich Observatory (RGO) covering the years 1874-1975 as their "standard" observer. Because Wolf and successor Alfred Wolfer observed from 1848 through 1928 with overlap 1876-1893, their combined observations can serve as a "backbone" for the determination of $k$-factors for other observers overlapping with them before the RGO series begins. H\&S determined that the ratio between $k$-factors for Wolf and Wolfer was 1.021 , i.e., that both observers saw very nearly the same number of groups. And herein lies the problem.

Because of extensive travel and other duties, Wolf from the 1860s until his death in 1893 exclusively used a small, handheld telescope, while Wolfer used a larger $80 \mathrm{~mm}$ Fraunhofer telescope similar to what Wolf used earlier. Both telescopes still exist (Fig. 4) and are even in use today by Thomas Friedli in Belp, near Bern, continuing the Wolf tradition.

Figure 5 shows that for the time of overlap between Wolf and Wolfer, Wolfer counted (as appropriate for the larger telescope) 1.653 times as many groups as Wolf, and not only 


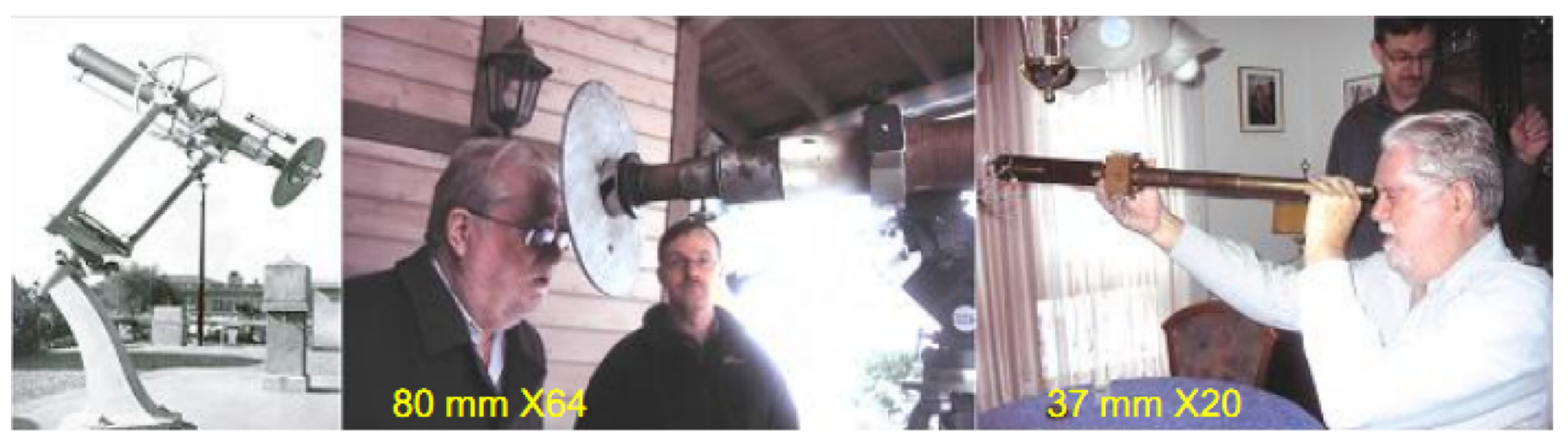

Fig. 4. Left: the $80 \mathrm{~mm}$ Fraunhofer refractor used since 1855 by Wolf and successors. Center: the same telescope in use today by Thomas Friedli (person at right). Right: the $37 \mathrm{~mm}$ portable telescope used by Wolf since the mid-1860 s.
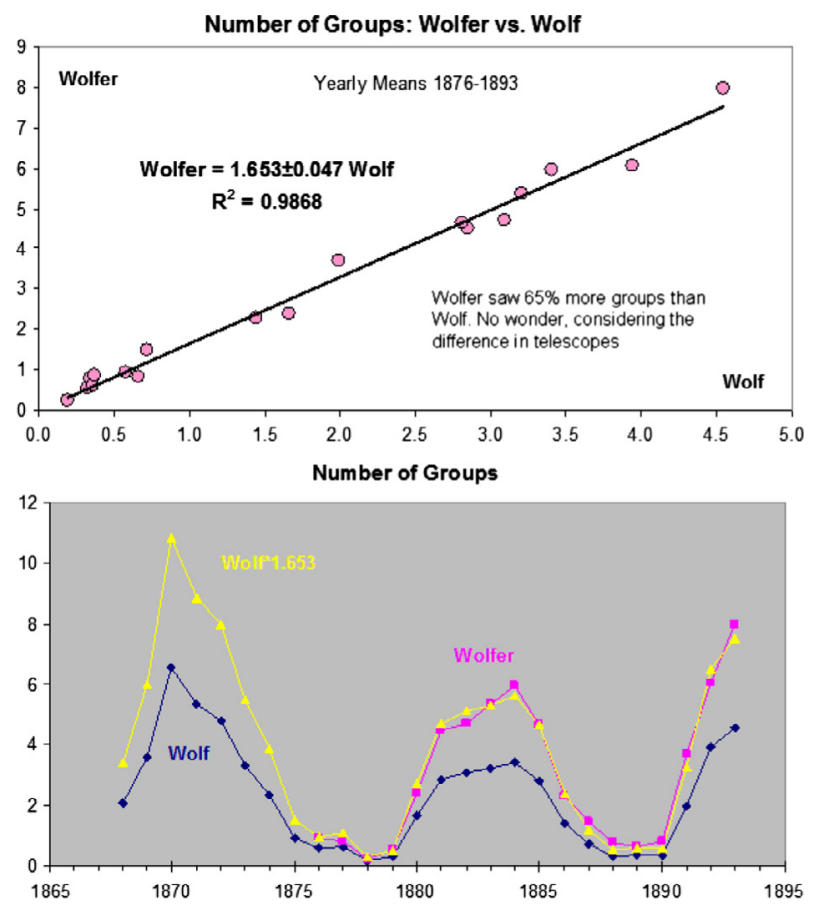

Fig. 5. Top: number of groups observed by Wolfer compared to the number observed by Wolf. Bottom: Applying the $k$-factor of 1.653 makes Wolf's count (blue) match (yellow) Wolfer's count (pink).

1.021 times as determined by H\&S. This discrepancy is the main cause of the 1885 discontinuity in the GSN/SSN ratio. We can understand the reason why Wolf saw so few groups by considering that the small spots making up groups with Zürich classifications $a$ and $b$ (groups containing spots without penumbra) are usually not visible in Wolf's small telescope. Such groups make up about a half of all groups.

Using the Wolf-Wolfer composite record as a backbone, we can now confidently determine $k$-factors for 22 mutually overlapping observers stretching back to Schwabe and forward to Brunner and construct a composite series. As Figure 6 shows there is now no significant difference between the GSN and the SSN. So, with only two adjustments: $\sim 20 \%$ for SSN before 1945 and $\sim 50 \%$ for GSN before 1885, the discrepancy is resolved. The issue of the very early data, say before 1825 , is still open and is the target for the next SSN Workshop, but if people can accept the current series without adjustments, then they might also accept that we, for now, assume that there are no further adjustments warranted for the early data.

\section{Geomagnetic calibration of sunspot numbers}

Wolf (1852) discovered a beautiful connection between sunspots and the diurnal variation of the Earth's magnetic field. He marvelled "Who would have thought just a few years ago about the possibility of computing a terrestrial phenomenon from observations of sunspots", and he at once realized that such a relation could be used as an independent check on the calibration of the sunspot number. Today we understand the physics of that connection and can fully validate Wolf's assertion. Solar Far Ultraviolet (FUV) radiation creates and maintains the conducting E-layer in the ionosphere. Thermal winds driven by solar heating move the charges across the Earth's magnetic field setting up an ionospheric dynamo with currents generating magnetic effects observable on the ground. As the Earth rotates under the currents (which are fixed in direction to the Sun) a characteristic diurnal perturbation of the geomagnetic field is readily observed (discovered by Graham in 1722). The diurnal variation is best seen in the East component of the geomagnetic field. The $10.7 \mathrm{~cm}$ microwave flux from the Sun is a good proxy for the FUV flux and Figure 7 shows how well the amplitude of the diurnal variation tracks 10.7, thus validating Wolf's procedure using modern data.

The Figure also shows the "equivalent" 10.7 flux calculated from the observed range (average of nine stations) of the diurnal variation for many solar cycles before the advent of the flux radiometers. It is clear that the well-understood physics of causes of the diurnal variation of the geomagnetic field provides a reliable way of assessing the past variation of solar flux, and hence the magnetic activity responsible for it; a variation for which the sunspot number is a proxy. Careful application of this method fully supports the two adjustments of the sunspot series described above based on the diurnal variation as observed since the 1780 s.

\section{Solar wind properties in the past}

Direct in-situ observation of the solar wind goes back 50 years and it was clear from even the earliest data that geomagnetic activity (separate from and superposed on the regular diurnal variation just discussed) was directly controlled by the expanding solar atmosphere - by the expansion speed and by the strength and direction of the magnetic field dragged out from the Sun. Recent research (Svalgaard \& Cliver 2005, 2007, 2010; Lockwood \& Owens 2011) has shown that it is possible to infer the solar wind speed and the magnetic field strength from suitable, newly defined, indices of geomagnetic activity 


\section{Comparison Composite Groups and Scaled Zurich SSN}

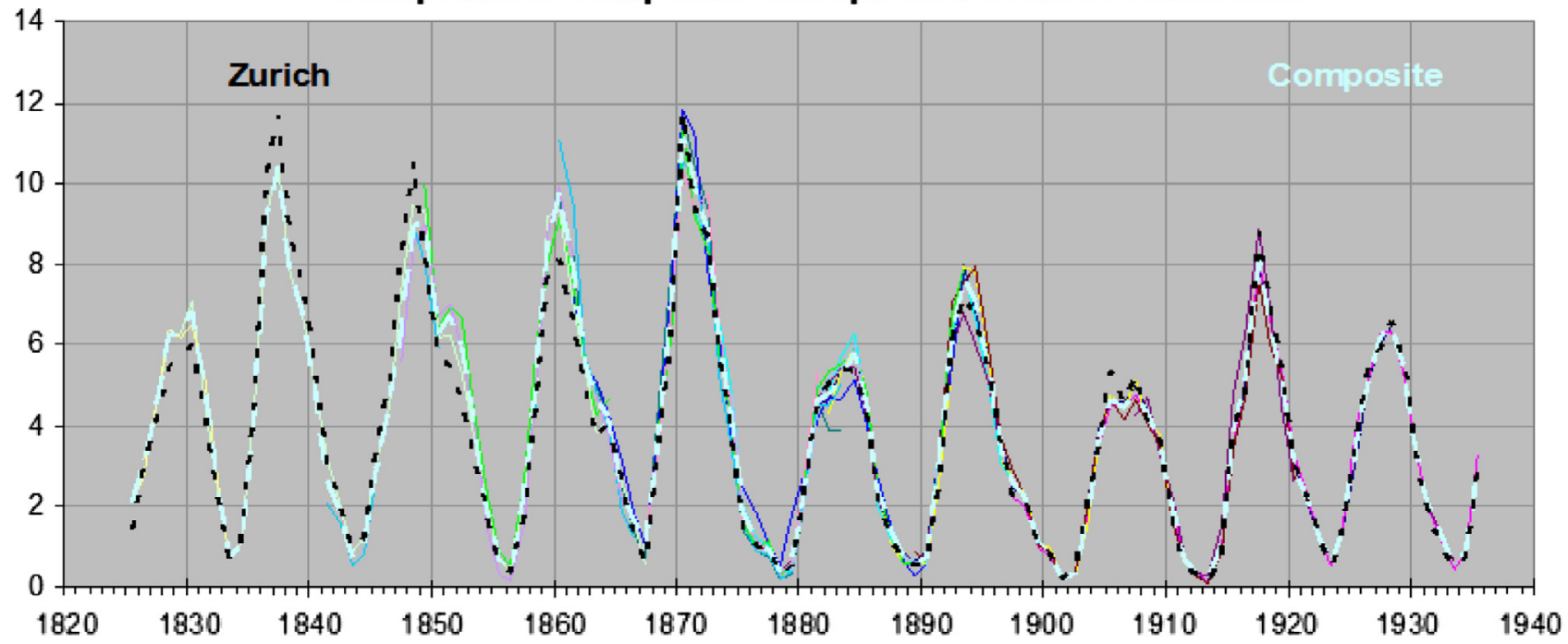

Fig. 6. Different colors (hard to see because they fall on top of each other) show the individual contributions to the composite (bright cyan). The black dashed curve is the Zürich SSN/12 (to convert it to number of groups).

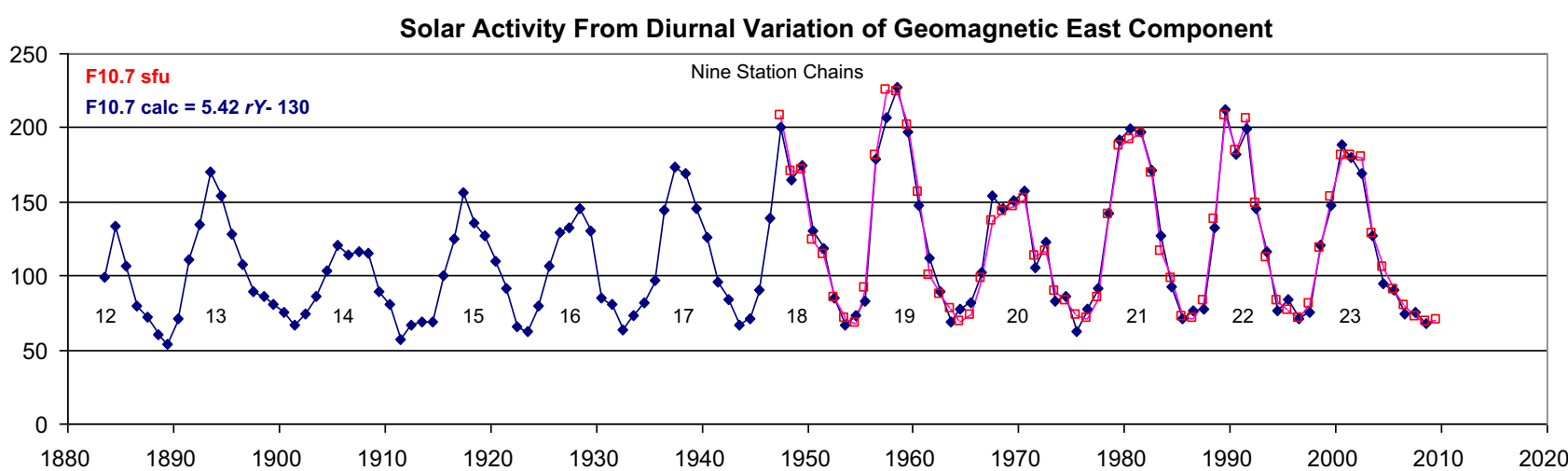

Fig. 7. 10.7 Flux correlation with the range, $r Y$, of the diurnal variation of East Component.

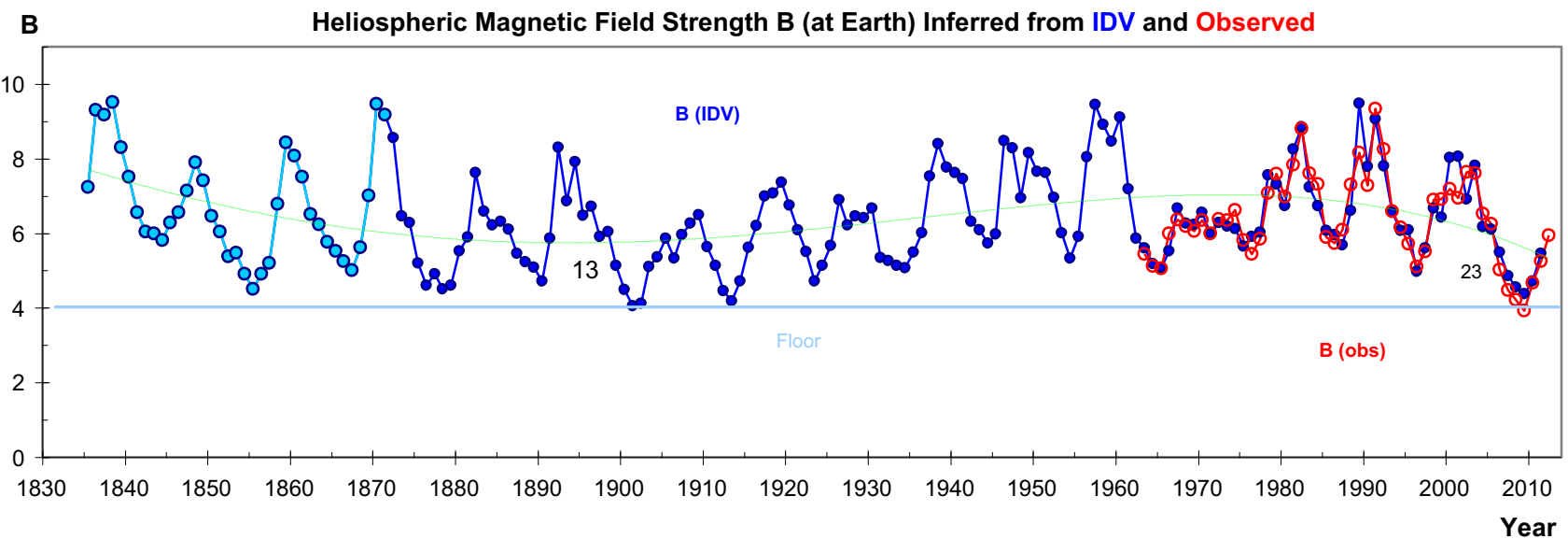

Fig. 8. Heliospheric magnetic field strength $B$ at Earth inferred from the $I D V$ geomagnetic index (blue) and observed by spacecraft (red). Data before 1872 (light blue) are preliminary and can be improved by adding more 19th observatory data.

that have been found to respond to different combinations of these solar wind parameters, allowing the influence of each to be separately extracted and calibrated by comparison with the space-based data measured near the Earth, effectively inverting the "response function" of the Earth to the solar wind. Figure 8 shows one result of this inversion.

Data exist to carry this inversion back to the earliest systematic observations of the geomagnetic field in the 1830s. We find 


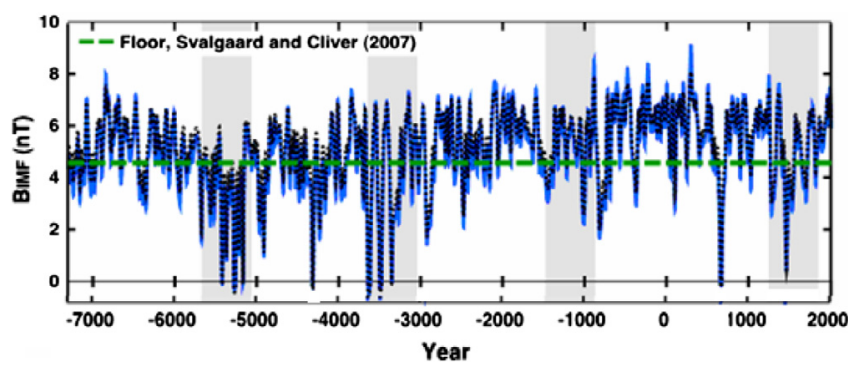

Fig. 9. Heliospheric magnetic field strength at Earth inferred from the cosmic ray record (Steinhilber et al. 2010). The deep excursions to zero or even un-physical negative values are not understood and may be artifacts caused by too aggressive extrapolation from modulation potential to field strength. This is presently an open and controversial issue.

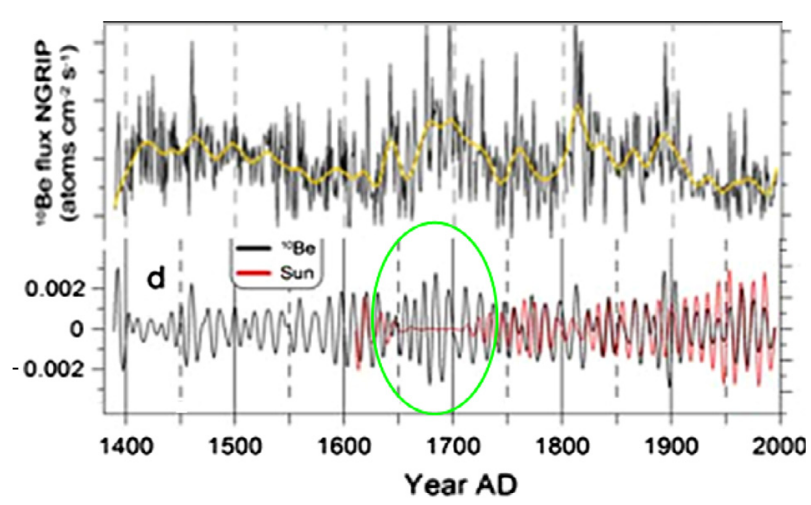

Fig. 10. Top: ${ }^{10} \mathrm{Be}$ flux from the NGRIP ice core (Greenland). Bottom: the ${ }^{10} \mathrm{Be}$ data band pass filtered at $8-16$ years (Berggren et al. 2009). The red curve is the filtered GSN series.

that the reconstruction of the solar wind is consistent with the reassessment of the sunspot number series described above, in particular that there also does not seem to have been a Modern Grand Maximum in solar wind parameters. Another finding of interest is that even during periods of extremely low solar activity (e.g., the years 1901-1902, 2008-2009) the solar wind is still present having a respectable magnetic field of about 4 nT. An important research issue at present is whether this minimum state of solar magnetic activity, a "floor", is a general feature, at all times (Schrijver et al. 2011). Recent work by Owens et al. (2012) suggests that "even a steady decline in sunspot number may result in a plateau in the Open Solar (magnetic) Flux".

\section{The cosmic ray record}

Cosmic ray particles reaching the Earth are mostly produced outside the solar system during supernova explosions and subsequent acceleration in the Galaxy. Two time-varying magnetic "shields", the solar magnetic field and the geomagnetic field, modulate the cosmic ray flux. The weaker these fields, the higher is the cosmic ray intensity near the surface of the Earth. Ionization chambers and neutron monitors have directly monitored the intensity of cosmic radiation since the $1930 \mathrm{~s}$ (Steinhilber et al. 2012). Before that, no direct measurements exist, and cosmogenic radionuclides, are used as a proxy for cosmic radiation, especially ${ }^{10} \mathrm{Be}$ and ${ }^{14} \mathrm{C}$, produced by cosmic rays colliding with atmospheric nitrogen and oxygen. Thus, the production rates of these nuclides are related to the flux of the incoming cosmic rays. As with the sunspot number, there are issues with the proper calibration of the cosmic ray proxies. What are measured are not variations of the production rate, but of the deposition rate, which in addition to the incoming flux also depend on atmospheric circulation and climate in general. Inversions of the ionization chamber data to extract the strength of the solar magnetic field are discordant with inversions of the neutron monitor data and with the result of the geomagnetic constructions. This issue will eventually be resolved and a special ISSI workshop toward this goal is ongoing (Svalgaard et al. 2011).

Of special interest is the cosmic ray record of so-called "Grand Minima", like the Maunder Minimum. The solar magnetic field (expressed as the near-Earth solar wind magnetic field) extracted from the cosmic ray record falls to zero or at times is un-physically negative during Grand Minima (Fig. 9) while at the same time a vigorous solar cycle modulation of the cosmic ray flux is observed (e.g., Berggren et al. 2009, Fig. 10) indicating to this author that significant solar magnetic field was present.

Similarly, observations of the spicule forest (the "red flash") during the total solar eclipses in 1706 and 1715 seem to require the presence of bright network structures, and thus of substantial solar photospheric and chromospheric magnetism during at least the last decades of the Maunder Minimum (Foukal \& Eddy 2007).

The very long cosmic ray record (when calibrated and understood correctly - including the effect of solar cosmic rays) provides the necessary material for statistical studies of the frequency and features of extremes of solar activity (the " 500 -year floods"). The first order of business is to understand why the variations are discordant compared to other solar indicators the past 400 years. This effort is ongoing and the results are not yet in sight.

\section{Predicting solar activity}

At this point in time it has become of great practical and societal importance to predict solar activity and space climate, rather than just recording them, e.g., for planning mitigation of the effects of extreme solar events. The NASA/NOAA international Panel for Predicting the Solar Cycle examined 75 "predictions" of the current Cycle 24, basically covering the full spectrum of possible outcomes centered on the climatological mean, from extremely small to extremely large (Pesnell 2012). The Panel ended up (barely) endorsing the "precursor" methods as the most promising where some property of the Sun near minimum is used as a predictor of the following cycle. On physical grounds, the magnitude of the polar fields of the Sun seems to be a good candidate as a precursor as it is thought that the polar fields act as a "seed" for the dynamo producing the next cycle (e.g., Jiang et al. 2007). Leading up to the minimum in 2008, the polar fields were the weakest ever observed (since the invention of reliable solar magnetographs in the 1950s) prompting Svalgaard et al. (2005) and Schatten (2005) to predict that cycle 24 would be "the smallest cycle in a hundred years". This prediction has, so far, held up well, providing (together with predictions using other precursors, such as the geomagnetic $a a$-index) a successful test of the precursor notion. Figure 11 compares the polar fields and the size of the next cycle for the past several cycles. 

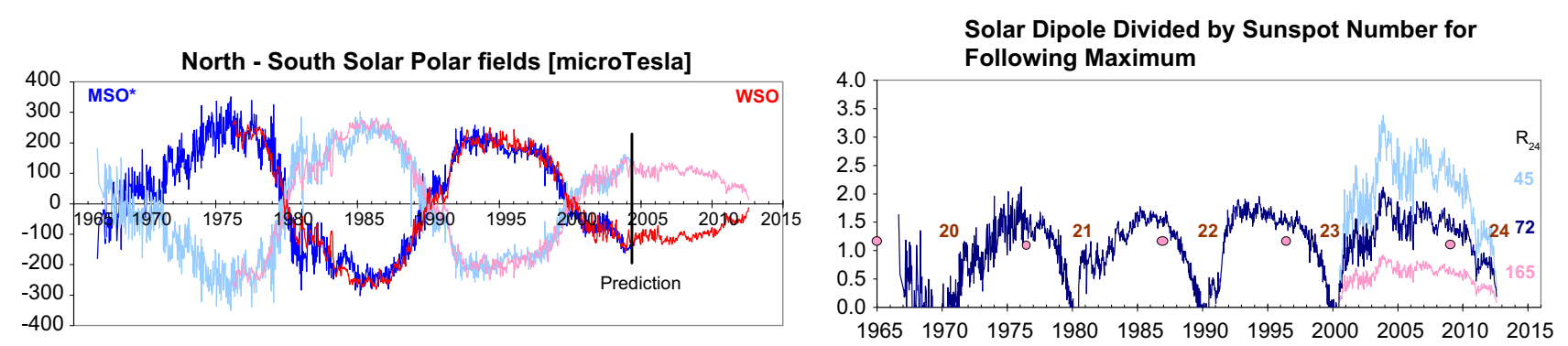

Fig. 11. Left: the axial magnetic "dipole moment" of the Sun, defined as the difference between the (signed) magnetic field strength near the North Pole and near the South Pole, for the past four solar cycles. To facilitate comparison of cycles, a "ghost" mirror image is also plotted. Data from Mount Wilson Observatory (blue) has been scaled to match observations from Wilcox Solar Observatory (red). Right: the polar field variation at the left scaled by the maximum smoothed sunspot number for the next cycle suggesting the same variation for each cycle, i.e., that the cycle maximum is controlled by the polar fields at the preceding minimum. For Cycle 24, the maximum was not known, but a very high value (165) or a very low value (45) does not fit the pattern. A maximum size of 72 for Cycle 24 seems to be the just right "Goldilocks" value.

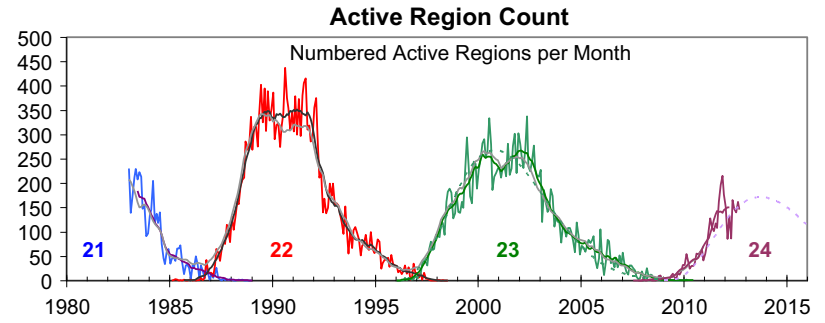

Fig. 12. Numbered active regions within $70^{\circ}$ of $\mathrm{CM}$ per month. Different cycles are plotted with different colors. The predicted Cycle 24 is shown as the dashed purple curve. We are very close to a drawn out solar maximum at this time of writing.

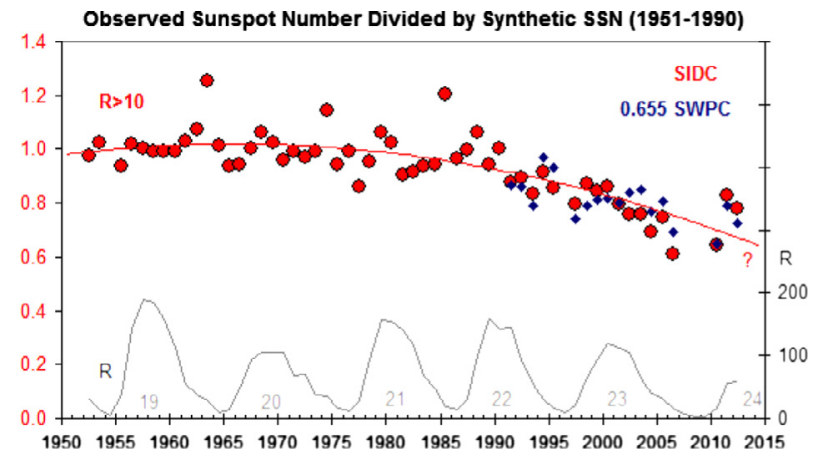

Fig. 13. The observed SSN divided by a synthetic SSN computed from a polynomial fit to the 10.7 flux over the interval 1951-1990. Red points are SIDC sunspot numbers while blue points are SWPC (NOAA) sunspot numbers scaled down by the their average $k$-factor of 0.655 . Only years when the SSN was above 10 are included. Since $\sim 1990$ the observed SSN is progressively lower for a given 10.7 flux.

Figure 12 shows how the prediction is doing. The quantity plotted is the total number of active regions per month on the disk within $70^{\circ}$ of Central Meridian (which is on average 2.25 times the sunspot number).

\section{Recent changes in the sun?}

Historically all solar indices have been closely correlated as they all derive from the same source: the variable magnetic field. In fact, the various reconstructions of past (and predictions of future) activity all rely on the implicit assumption that the correlations stay the same over time. This is likely to be true for indices that have a close physical connection, like the 10.7 and UV fluxes, but is not given a priori for correlations that are more indirect, e.g., involving the sunspot number: the processes creating visible sunspots are varied and not fully understood. And indeed, while there has long been a stable relationship between the 10.7 flux and the sunspot number, allowing one to calculate or map one from the other, that relationship has steadily deteriorated in the past decade to the point where the sunspot number for a given flux has decreased by about a third (Fig. 13).

A similar decrease of the sunspot number for a given amount of magnetic flux in the plages surrounding active regions has occurred as well as a decrease of the number of spots per active region. This is unprecedented in the observational record. We interpret this decrease as a loss of primarily the small spots (Clette \& Lefèvre 2012).

Observations by Livingston and Penn since 1998 until the present show that the average magnetic field in sunspots has steadily decreased by $25 \%$ (Livingston et al. 2012), regardless of the fact that we are now again at the maximum of a solar cycle, so there has not been a solar-cycle-related reversal of the trend. Since their magnetic fields cool sunspots, a decreasing field means that sunspots are getting warmer and that their contrast with the surrounding photosphere is getting smaller, making the spots harder to see. There is a minimum field strength in visible spots of about 1500 Gauss $(0.15 \mathrm{~T})$ and as that $1500 \mathrm{G}$ threshold is approached, magnetic fields appear at the solar surface which do not seem to form dark sunspots or pores. Owens et al. (2012) suggest that the photospheric flux emergence in such cases may take place in flux tubes with field too weak, or of too small a diameter, to form sunspots, citing Spruit (1977). The observed distribution of number of spots vs. field strength has been shifting steadily toward that limit. If, and that is a big $I F$, this trend continues, the number of visible spots in the next cycle (and perhaps beyond) may fall to values not seen since the Maunder Minimum, but without dramatic changes in the emerging magnetic flux. Without the dark spots, Total Solar Irradiance might even be a bit higher. It is not clear what this will mean for the impact of solar activity on the Earth's environment, if any, but it portends exciting times for solar physicists. 


\section{Discussion}

Our technological civilization has reached a point where solar activity and its prediction on all times scales have become significant factors in maintaining and safekeeping of the technological infrastructure, both on the ground and in space. New capabilities, in instrumentation, deployment, computer storage and power, and - last, but not least - increased awareness promise progress toward predictive improvements. On the other hand, if the Sun is moving into a new regime of lower activity, a period of uncertainty may make life hard for the forecaster. The possible, recent reassessment of past solar activity should provide a better benchmark for theoretical modeling to meet. If the discrepancy between the sunspot number and other solar indices continues, it may be that the SSN, for a while, is no longer a good measure of solar activity and forecasters (and users) may be forced to rely on other indices for operational use. This does not mean that we should stop deriving the sunspot number from the usual visual observations; on the contrary, the evolution of the SSN must be followed closely in order to provide a continuing basis for assessing the historical record.

Acknowledgements. The author thanks Ed Cliver for serving as a long-suffering sounding board for my wilder ideas. I also thank Phil Scherrer at Stanford for support and I have benefited from participation in the International Teams in Space Science (ISSI) Workshop on "Long-term reconstruction of Solar and Solar Wind Parameters" and from the Sunspot Number Workshops http://ssnworkshop. wikia.com/wiki/Home

\section{References}

Berggren, A.-M., J. Beer, G. Possnert, A. Aldahan, P. Kubik, M. Christl, S.J. Johnsen, J. Abreu, and B.M. Vinther, A 600-year annual ${ }^{10} \mathrm{Be}$ record from the NGRIP ice core, Greenland, Geophys. Res. Lett., 36, L11801, DOI: 10.1029/2009GL038004, 2009.

Clette, F., and L. Lefèvre, Are the sunspots really vanishing? Anomalies in solar cycle 23 and implications for long-term models and proxies, J. Space Weather Space Clim., 2, A06, 2012.

Cliver, E.W., and L. Svalgaard, The 1859 solar-terrestrial disturbance and the current limits of extreme space weather activity, Sol. Phys., 224, 407, 2004

Eddy, J.A., The Maunder Minimum, Science, 192 (4245), 1189, DOI: 10.1126/science.192.4245.1189, 1976.

Foukal, P., and J. Eddy, Did the sun's prairie ever stop burning? Sol. Phys., 245, 247, DOI: 10.1007/s11207-007-9057-8, 2007.

Hoyt, D.V., K.H. Schatten, and E. Nesme-Ribes, The one hundredth year of Rudolf Wolf's death: do we have the correct reconstruction of solar activity? Geophys. Res. Lett., 21 (18), 2067, DOI: 10.1029/94GL01698, 1994.

Jiang, J., P. Chatterjee, and A.R. Choudhuri, Solar activity forecast with a dynamo model, Mon. Not. R. Astron. Soc., 381, 1527, DOI: 10.1111/j.1365-2966.2007.12267.x, 2007.
Livingston, W., M.J. Penn, and L. Svalgaard, Decreasing sunspot magnetic fields explain unique $10.7 \mathrm{~cm}$ radio flux, Astrophys. $J$., 757 (1), L8, DOI: 10.1088/2041-8205/757/1/L8, 2012.

Lockwood, M., and M.J. Owens, Centennial changes in the heliospheric magnetic field and open solar flux: the consensus view from geomagnetic data and cosmogenic isotopes and its implications, J. Geophys. Res., 116, A04109, DOI: 10.1029/2010JA016220, 2011.

National Research Council, NRC, Severe Space Weather Events Understanding Societal and Economic Impacts: A Workshop Report, Washington, DC, The National Academies Press, 2008.

Owens, M.J., I. Usoskin, and M. Lockwood, Heliospheric modulation of galactic cosmic rays during grand solar minima: past and future variations, Geophys. Res. Lett., 39, L19102, DOI: 10.1029/2012GL053151, 2012.

Pesnell, W.D., Solar cycle predictions (invited review), Sol. Phys., The Sun, 360, DOI: 10.1007/s11207-012-9997-5, 2012.

Schaefer, B., Visibility of sunspots, Astrophys. J., 411, 909, 1993.

Schatten, K.H., Fair space weather for solar cycle 24, Geophys. Res. Lett., 32, L21106, DOI: 10.1029/2005GL024363, 2005.

Schrijver, C.J., W.C. Livingston, T.N. Woods, and R.A. Mewaldt, The minimal solar activity in 2008-2009 and its implications for long-term climate modeling, Geophys. Res. Lett., 38, L06701, DOI: 10.1029/2011GL046658, 2011.

Spruit, H.C., Heat flow near obstacles in the solar convection zone, Sol. Phys., 55, 3, DOI: 10.1007/BF00150871, 1977.

Steinhilber, F., J.A. Abreu, J. Beer, and K.G. McCracken, Interplanetary magnetic field during the past 9300 years inferred from cosmogenic radionuclides, J. Geophys. Res., 115 (A1), A01104, DOI: 10.1029/2009JA014193, 2010.

Steinhilber, F., J.A. Abreu, J. Beer, et al., 9,400 years of cosmic radiation and solar activity from ice cores and tree rings, Proc. Nat. Acad. Sci., 109 (16), 5967, 2012.

Svalgaard, L., and E.W. Cliver, The IDV index: its derivation and use in inferring long-term variations of the interplanetary magnetic field strength, J. Geophys. Res., 110, A12103, DOI: 10.1029/2005JA011203, 2005.

Svalgaard, L., and E.W. Cliver, Interhourly variability index of geomagnetic activity and its use in deriving the long-term variation of solar wind speed, J. Geophys. Res., 112, A10111, DOI: 10.1029/2007JA012437, 2007.

Svalgaard, L., and E.W. Cliver, Heliospheric magnetic field 18352009, J. Geophys. Res., 115, A09111, DOI: 10.1029/2009JA015069, 2010.

Svalgaard, L., M. Lockwood, and J. Beer, Long-term reconstruction of solar and solar wind parameters, ISSI team 233, http:// www.leif.org/research/svalgaard_ISSI_Proposal_Base.pdf, 2011.

Waldmeier, M., Die Beziehung zwischen der Sonnenflecken-relativzahl und der Gruppenzahl, Astr. Mitteil. Eidgn. Sternw Zürich, Nr. 285, 1968.

Wolf, J.R., Neue Untersuchungen über der Periode der Sonnenflecken und ihre Bedeutung, Mittheil. der naturforsch. Gesellshaft in Bern, Nr. 245, 1852.

Cite this article as: Svalgaard L: Solar activity - past, present, future. J. Space Weather Space Clim., 2013 , 3, A24. 